\title{
Characterization of Heterogeneous Response of Al Bicrystal Subject to Micro Scale Laser Shock Peening
}

\author{
S. Vukelic • I.C. Noyan • J.W. Kysar • Y.L. Yao
}

Received: 9 February 2010 / Accepted: 7 June 2010 /Published online: 23 July 2010

(C) Society for Experimental Mechanics 2010

\begin{abstract}
This letter shows the ability to perform characterization of the strain field in an aluminium bicrystal subject to plane strain condition induced by micro scale laser shock peening. Intensity contrast method, previously used in topographic measurements of strain fields in thin films is employed here. Our results show that this method is applicable for measurements of the plastically deformed bulk materials. Moreover, heterogeneous response of the grain boundary is observed as periodic nature of deformation.
\end{abstract}

Keywords Laser · Bicrystal · Plasticity · X-ray diffraction . Intensity contrast method

The Laser Shock Peening (LSP) process introduces surface compressive residual stresses which improve material properties of various metals 1-3]. Its main advantages over conventional treatments is deeper shock wave penetration, as well as process flexibility which allows treatment of parts with complicated geometries. The high cost of high power lasers required for LSP led to the development of micro scale laser shock peening [4]

\footnotetext{
S. Vukelic $(\bowtie)$

Mechanical Engineering Department, Bucknell University, Pennsylvania, PA 17837, USA

e-mail: sv004@bucknell.edu

I.C. Noyan

Program in Materials Science,

Department of Applied Physics and Applied Mathematics, Columbia University,

New York, NY 10027, USA

J.W. Kysar (SEM Member) • Y.L. Yao

Mechanical Engineering Department, Columbia University,

New York, NY 10027, USA
}

( $\mu$ LSP). This process can be used to improve fatigue life and wear resistance of miniature devices such as micro switches, MEMS, etc. Since the diameter of the laser spot used during $\mu \mathrm{LSP}$ is of the same order of magnitude as the grain size of most materials undergoing the process, the effects of sample anisotropy [5-7] and heterogeneity [8] have to be explicitly taken into account in the analysis of the residual field formation.

X-ray topography is an excellent non-destructive method for characterization of defects and strain fields in thin films. With this technique the intensity contrast produced by local strain gradients or lattice rotations is used to characterize elastic and plastic deformation fields. The Intensity contrast method has been used for the characterization of metallic thin films processed with $\mu \operatorname{LSP}[9,10]$.

The goal of the current study is to employ the intensity contrast method to characterize plastic deformation induced in bicrystal aluminium samples subjected to $\mu \mathrm{LSP}$. We have been able to observe the effects of heterogeneity as a consequence of shock wave interaction with the grain boundary, and the distribution of plastic deformation throughout the material.

An aluminum bicrystal grown from the melt with the modified Bridgman technique was used. The grain boundary between the crystals was a CSL $\Sigma 43$ symmetric tilt-type with the [110] direction parallel to the tilt axis of the adjoining grains. The surfaces of the individual crystals deviated about $9.5^{\circ}$ and $2.5^{\circ}$ from the ideal (110) and (001) surfaces, respectively. These orientations were chosen because, if the loading line is placed along $\langle 110\rangle$ direction, an approximate plane strain deformation will be induced [11, 12]. A frequency tripled Q-switched Nd:YAG laser with wavelength $\lambda=355^{\circ} \mathrm{nm}$ in $\mathrm{TEM}_{00}$ mode was used for the 
$\mu$ LSP experiments. The beam diameter was $6 \mu \mathrm{m}$ and pulse duration was $50 \mathrm{~ns}$ with approximate laser intensity of $4 \mathrm{GW} / \mathrm{cm}^{2}$. Distilled water was used as a confining medium and laser shocks were applied with $25 \mu \mathrm{m}$ spacing as an approximate line loading parallel to the grain boundary and about $80 \mu \mathrm{m}$ away from it into the (110) crystal. More details about experimental setup can be found in $[4,8]$.

$\mathrm{X}$-ray scanning microtopography data was collected on a diffractometer using focusing capillary optics on the X-20A beamline of the National Synchrotron Light Source at Brookhaven National Laboratory. Monochromatic synchrotron light at $8 \mathrm{keV}$ energy $(\lambda=1.5406 \AA)$ was used [13]. The $\mathrm{x}$-ray spot diameter was measured with the knife edge technique and appeared to be elliptical with major and minor axes of about $20 \mu \mathrm{m}$ and $10 \mu \mathrm{m}$, respectively. The sample was mounted on a high resolution $x-y$ translation stage with $1 \mu \mathrm{m}$ resolution. Integrated intensity variation as a function of position was obtained with step sizes of $20 \mu \mathrm{m}$. The region near the grain boundary in both crystals was mapped using $\mathrm{Al} 002$ and 220 reflections, respectively. A simple sphere of confusion error optimization technique [13] was employed to minimize machine errors when switching between crystals. The experimental setup is shown in Fig. 1.

Diffracted intensity variation in the strained regions can be caused by three different mechanisms: lattice rotation, lattice dilatation, and the presence of lattice parameter gradients within the irradiated volume. Both lattice rotation and lattice dilatation move the material away from the Bragg condition (assuming monochromatic radiation and fixed incident beam and detector angles), thus causing a drop in diffracted intensity. Local lattice parameter or strain gradients change the mode of scattering from fully dynamical to partly kinematical, and increase the band-pass of the crystal. This causes an increase of the diffracted intensity for divergent incident beams.

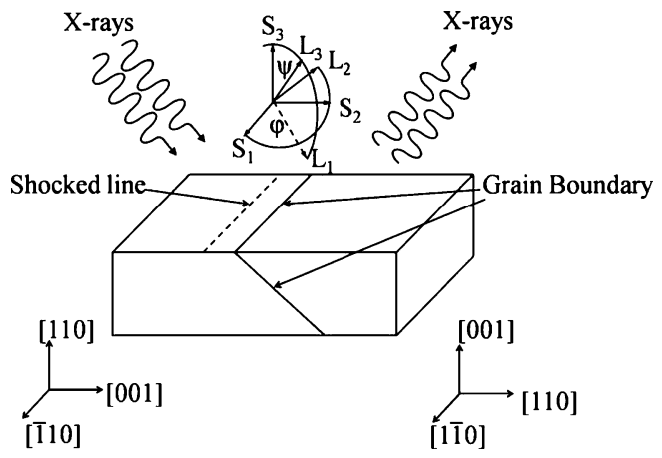

Fig. 1 Experimental geometry
During processing $\mu$ LSP laser pulses cause shock waves which induce plastic deformation in the target material. In addition, we expect dislocations to pile-up in the vicinity of the grain boundary of the aluminium bicrystal. This is seen in Fig. 2, where the integrated intensity is observed to increase along the shock line. The width of the region is approximately $80 \mu \mathrm{m}$ which is consistent with EBSD results [8], suggesting that the intensity contrast method is applicable for qualitative characterization of plastically deformed bulk single and bicrystals.

Although laser shocks are placed very close to the grain boundary, a rise in integrated intensity is observed only in the (110) crystal. This can be explained as an inability of the slip to transmit through the grain boundary. After the resolved shear stress reaches critical value, dislocation motion on the slip planes is activated. However, the grain boundary acts as an obstacle to dislocation motion so dislocations pile up. Thus, plastic slip flows under compressive $\mu$ LSP loading in each crystal until it reaches the grain boundary, which inhibits the plastic slip. This can be seen in Fig. 3 where fullwidth-at-half-maximum (FWHM) is depicted as a function of spatial position relative to the grain boundary. The plastic deformation causes x-ray peak profile broadening and from the Fig. 3 it can be seen that no deformation is observed in (001) crystal. Figure 3 also exhibits periodic variations in the FWHM with distance. The largest peak is at the position of the laser shock line and it decays towards the interior of the (110) crystal. On the other hand, no increase in FWHM is observed in (001) crystal (on the left side of the graph), suggesting that the grain boundary impedes propagation of the plastic deformation. It should be also noted that the integrated intensity has minimum value exactly at the grain boundary. That is due to machine error as at that point orientation changes and diffracted signal is weak resulting in diminished value of the integrated intensity. As mentioned above, $\mu$ LSP induces shock waves that propagate into the target material and cause plastic deformation. One possible physical explanation for the observed material response is that periodic behaviour emerges from the interference between waves reflected from the grain boundary with those originally propagating in the direction of the grain interior. However, this matter requires more theoretical attention and it will be subject of the future study.

In summary, topographic x-ray microdiffraction study of an aluminium bicrystal subject to $\mu$ LSP has shown that intensity contrast method, previously used for characterization of thin films is applicable for qualitative strain measurements of plastically deformed bulk specimens. 
Fig. 2 Integrated intensity in the (110) crystal; (a) topographic representation of the portion of the shocked area; (b) various cross-sections of the integrated intensity
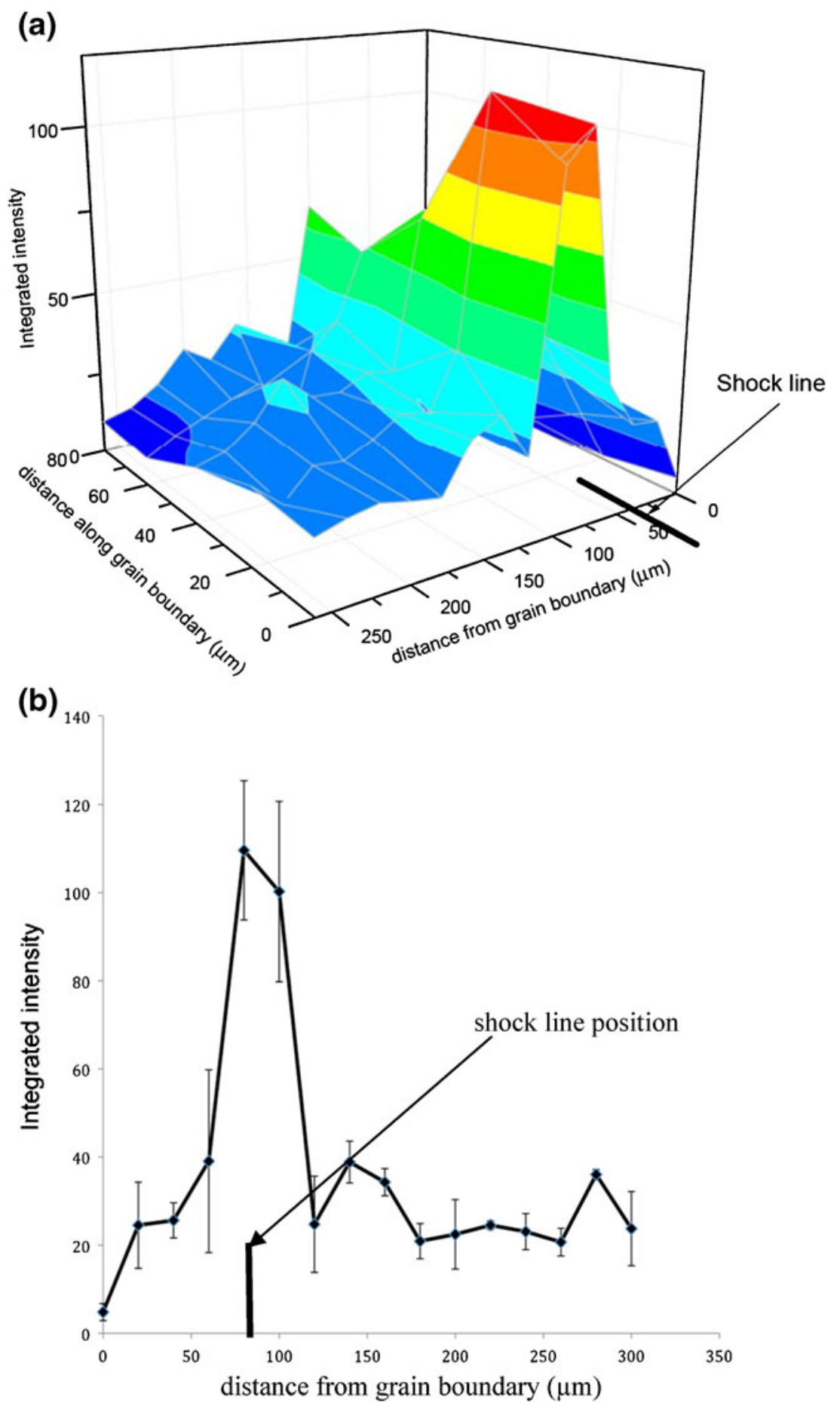

Thus, the intensity contrast method has potential to be used as a non-destructive characterization tool for plastically deformed metallic micro components. The data suggests that the grain boundary acts as an obstacle to the propagation of the plastic deformation.

This work is supported by NSF CMMI-0500239, CMMI-0620741, DMR-0706058, CMMI-0826093 and by AFOSR FA9550-09-1-0048. The authors would like to thank Dr. Jean Jordan-Sweet of IBM Watson Research Center for assistance in using x-ray microdiffraction apparatus at the Synchrotron Light Source at Brookhaven National Laboratory. This work was supported partially by the MRSEC program of the National Science Foundation under Award No. DMR-0213574 and by 


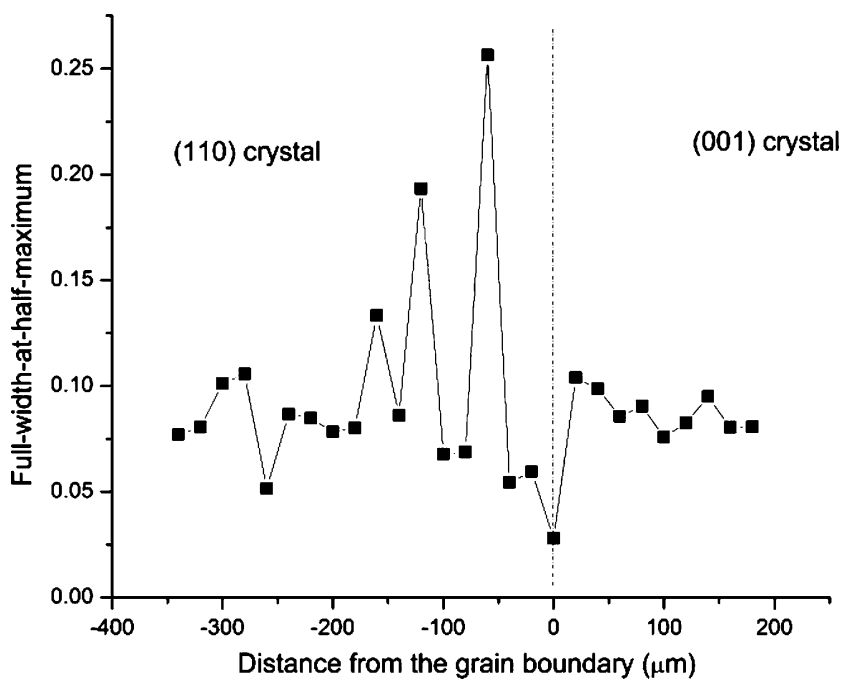

Fig. 3 Cross-sectional view of the full-width-at-half-maximum. Dashed line represents grain boundary between (110) and (001) grains

the New York State Office of Science, Technology, and Academic Research (NYSTAR) through the use of the MRSEC materials characterization facility.

\section{References}

1. Clauer AH, Holbrook JH (1981) Shock waves and high strain phenomena in metals-concepts and applications. New York, 675-702

2. Clauer AH, Lahrman DF (2001) Key Eng Mater 197:121142

3. Hammersley G, Hackel LA, Harris F (2000) Opt Lasers Eng 34:327-337

4. Zhang W, Yao YL (2002) J Manuf Sci Eng 124:369-378

5. Vukelic S, Wang Y, Kysar JW, Yao YL (2008) J Mech Mater Struct 4:89-105

6. Vukelic S, Wang Y, Kysar JW, Yao YL (2008) J Manuf Sci Eng 131:031015-1-031015-10

7. Wang Y, Kysar JW, Yao YL (2008) Mech Mater 40:100-114

8. Vukelic, S. Kysar, J.W., Yao, Y.L., (2007) Proceedings of the International Manufacturing Science and Engineering Conference (MSEC2007)

9. Zhang W, Yao YL, Noyan IC (2004) J Manuf Sci Eng 126:10 17

10. Zhang W, Yao YL, Noyan IC (2004) J Manuf Sci Eng 126:1824

11. Kysar JW, Gan YX, Mendez-Arzuza G (2005) Int J Plast 21:1481-1520

12. Rice JR (1987) Mech Mater 6:317-335

13. Noyan IC, Kaldor SK, Wang PC, Jordan-Sweet J (1999) Rev Sci Instrum 70:1300-1304 